\title{
Efimov States in a Bose-Einstein Condensate near a Feshbach Resonance
}

\author{
Eric Braaten, H.-W. Hammer, and M. Kusunoki \\ Department of Physics, The Ohio State University, Columbus, OH 43210, USA
}

(Dated: April, 2003)

\begin{abstract}
Recent experiments with Bose-Einstein condensates of ${ }^{85} \mathrm{Rb}$ atoms near a Feshbach resonance have produced evidence for a condensate of diatomic molecules coexisting with the atom condensate. It should also be possible to create condensates of the triatomic molecules predicted by Efimov coexisting with the atom and dimer condensates. The smoking gun for the trimer condensate would be oscillatory dependence of observables on the binding energy of the trimer. It may also be possible to deduce the existence of the trimer condensate from the spectra of the bursts of atoms and dimers created in the disappearance of the trimers.
\end{abstract}

PACS numbers: 03.75.Fi, 03.65.Ge, 21.45.+v

Keywords: Efimov states, Bose-Einstein condensates, Feshbach resonances

In 1925, Albert Einstein predicted that at sufficiently low temperature, an ideal gas consisting of noninteracting point-like identical bosons will undergo a phase transition to a Bose-Einstein condensate (BEC), in which all the particles are in the same quantum state 1]. The superfluid phase of liquid ${ }^{4} \mathrm{He}$, was for a long time the only physical system that could be interpreted as a BEC. The interpretation is complicated not only by the strong interactions between the atoms, but also by the high density of liquid ${ }^{4} \mathrm{He}$. In 1995, Bose-Einstein condensation was observed in dilute trapped gases of ${ }^{85} \mathrm{Rb},{ }^{7} \mathrm{Li}$, and ${ }^{23} \mathrm{Na}$ [2]. These systems are close to the ideal gas limit considered by Einstein. Although the interactions between the atoms give rise to large mean-field effects, they are still weak in the sense that quantum fluctuations around the mean field are small. Their strength is controlled by the diluteness variable $n a^{3}$, where $n$ is the number density and $a$ is the $s$-wave scattering length. Moreover, the atoms can be accurately approximated by point particles. This approximation is controlled by the diluteness variable $n \ell^{3}$, where $\ell=\left(m C_{6} / \hbar^{2}\right)^{1 / 4}, C_{6}$ is the van der Waals coefficient, and $m$ is the mass of the atoms.

Atoms can have a large scattering length $a \gg \ell$ if a diatomic molecule close to the 2-atom threshold creates a resonant interaction between pairs of atoms. In this case, there is the possibility of a qualitatively different BEC with pointlike constituents $\left(n \ell^{3} \ll 1\right)$, but strong resonant 2-body interactions $\left(n a^{3} \gtrsim 1\right)$. We will refer to such a system as a resonant $B E C$. In contrast to liquid ${ }^{4} \mathrm{He}$, the pointlike nature of the constituents in the resonant BEC provides hope that the behavior of the manybody system is determined by aspects of the few-body system that are dominated by the resonance.

The few-body problem for resonant atoms exhibits universal properties that do not depend on the details of the interactions responsible for the large scattering length. The most familiar of these properties in the case $a>0$ is the existence of a shallow 2-body bound state (hereafter called simply the dimer) whose binding energy in the limit $a \gg l$ is given by

$$
\left|E_{2}\right|=\hbar^{2} /\left(m a^{2}\right) .
$$

One particularly remarkable aspect of the 3-body system of resonant atoms is an accumulation of 3-body bound states (so called Efimov states) near threshold as $a \rightarrow \infty$ [3]. As $a$ increases, new Efimov states appear below the atom-dimer threshold at values of the scattering length that differ by multiples of 22.7: $a_{*}, 22.7 a_{*}, 515 a_{*}, \ldots$ In the limit $a=\infty$, there are infinitely many Efimov states with binding energies that differ by multiples of 515. There is convincing theoretical evidence that the ground state and excited state of the ${ }^{4} \mathrm{He}$ trimer are Efimov states [4]. Efimov states can be responsible for dramatic energy dependence of scattering observables in the 3-body sector. An interesting and challenging problem is to determine what effects they would have in a resonant BEC. The possibilities include self-trapped atom condensates 5] and mutually trapped atom and dimer condensates [6].

A convenient system for studying the resonant BEC is trapped atoms near a Feshbach resonance [7]. The scattering length $a$ is controlled by a magnetic field $B$ that can be tuned to arbitrarily large values. Studies of ${ }^{23} \mathrm{Na}$ condensates revealed large losses of atoms as $B$ was ramped towards or through the resonance where $a \rightarrow \pm \infty$ [8]. There have also been experiments on ${ }^{85} \mathrm{Rb}$ condensates near the Feshbach resonance at $155 \mathrm{G}[9]$. The most recent experiment has provided evidence for a molecular condensate coexisting with the atom condensate [10]. The molecular condensate consists of the dimers associated with the Feshbach resonance. The experiment also revealed other surprising features involving a burst of high energy atoms from the condensate and missing atoms.

In this Letter, we point out that it should also be possible in these experiments to create coexisting condensates of atoms, dimers, and Efimov trimers. We first summarize the relevant aspects of the recent ${ }^{85} \mathrm{Rb}$ experiment. We then describe how a condensate of Efimov trimers 
could be observed in similar experiments either through oscillations of observables with a frequency determined by the trimer binding energy or by the spectrum of the burst of atoms emitted by the trimer condensate.

The experiments of Ref. [10] began with condensates of $N \approx 16,000$ atoms at a magnetic field corresponding to a small scattering length $a_{\text {init }} \simeq 10 a_{0}$, where $a_{0}$ is the Bohr radius. The condensate was subjected to a timedependent magnetic field $B(t)$, which produced a scattering length $a(t)$ with the following time sequence: 1 . a first pulse up to a large scattering length $a_{\max } \approx 2500 a_{0}$ and then down to an intermediate value $a_{\text {hold }}, 2$. a variable holding time $\tau$ with constant $a=a_{\text {hold }}, 3$. a second pulse up to $a_{\max }$ and then down to a small scattering length $a_{\text {final }}$, 4. a slow ramp up to a large scattering length $\simeq 1400 a_{0}$ followed by a holding time to allow the condensate to expand, 5 . the sudden turn off of the magnetic field, which increased the scattering length through the resonance $a= \pm \infty$ to the zero-field value $-480 a_{0}$. The atoms were subsequently measured with a destructive imaging technique sensitive only to atoms with energies less than about $2000 \mathrm{nK}$. The time scales were 10's of $\mu \mathrm{s}$ for steps 1-3 and 5 and 1000's of $\mu$ s for step 4 . The observed atoms consisted of two components: (1) a remnant condensate of $N_{\text {remnant }}$ atoms with energies less than $3 \mathrm{nK}$, and (2) a burst of $N_{\text {burst }}$ atoms that were emitted from the condensate near the time of steps 1-3 and had a typical energy scale of $150 \mathrm{nK}$. Since these two numbers did not add up to $N$, there was also a third component: (3) $N_{\text {missing }}$ missing atoms.

The numbers $N_{\text {remnant }}$ and $N_{\text {burst }}$ were observed to be periodic functions of $\tau$ with frequency $\nu_{2}=\left|E_{2}^{\text {hold }}\right| / 2 \pi \hbar$, where $\left|E_{2}^{\text {hold }}\right|$ is the dimer binding energy when the scattering length is $a_{\text {hold }}$. This suggests that the system during step 2 contained coexisting atom and dimer condensates. The first pulse of $a(t)$ must have created the dimer condensate by a coherent transfer of atoms from the atom condensate. During the hold time, the two condensates would have evolved by phases that differ by the angle $2 \pi \nu_{2} \tau$. The second pulse would have induced coherent transfers of atoms between the two condensates. Atoms transferred from the dimer condensate could interfere with those in the preexisting atom condensate, resulting in an oscillatory dependence of $N_{\text {remnant }}$ on $\tau$ with frequency $\nu_{2}$. Assuming the mechanism for the burst involves the atom condensates, $N_{\text {burst }}$ should also oscillate as a function of $\tau$ with the same frequency $\nu_{2}$. One surprising result from the experiment is that the phase of $N_{\text {burst }}$ is shifted relative to that of $N_{\text {remnant }}$ by an angle that depends sensitively only on the fall time of the pulse in step 3. This feature seems to have been reproduced by the resonance field theory of Ref. 11].

A time-dependent scattering length also allows atoms to be transfered from the condensates to incoherent states of energetic atoms. We assume the evolution of $a(t)$ in step 4 was sufficiently adiabatic that very few atoms

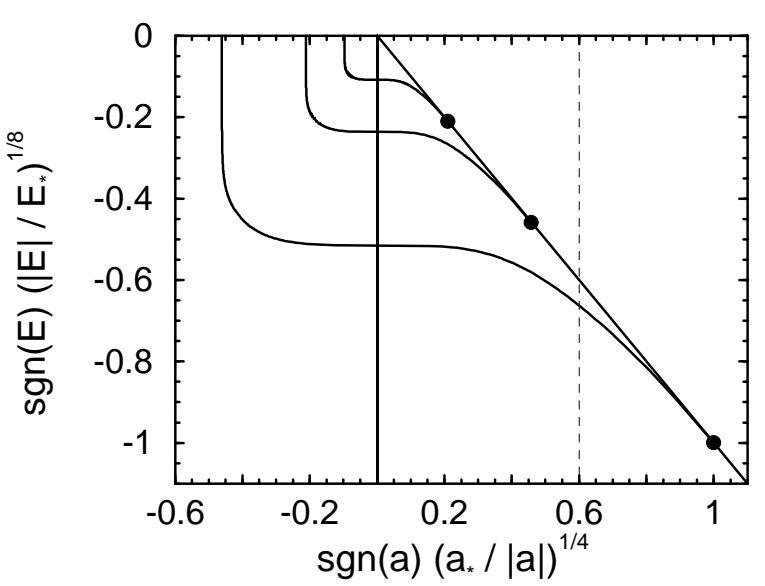

FIG. 1: The spectrum of Efimov states as a function of the scattering length $a\left(E_{*} \equiv \hbar^{2} /\left(m a_{*}^{2}\right)\right)$. The diagonal solid line is the atom-dimer threshold. The other solid lines are Efimov states. The vertical dashed line shows a typical $a>a_{*}$.

were transferred out of the condensates. We interpret the burst atoms and the missing atoms as the energetic atoms created by the changing scattering length during steps 1,3 and during step 5, repectively. The missing atoms would include atoms from the dissociation of the dimers in a dimer condensate due to the sweep through the Feshbach resonance in step 5.

The pulses that transfer atoms coherently to a dimer condensate could also transfer them coherently to a condensate of Efimov trimers. The existence of the trimer condensate could be verified by observing oscillatory effects involving the trimer binding energy $\left|E_{3}\right|$. When the scattering length $a$ is large, Efimov theory predicts $\left|E_{3}\right|$ in terms of $a$ and a single 3-body parameter that is insensitive to variations in $a$ [3, 4, 12]. A convenient choice for this 3-body parameter is the critical value $a_{*}$ of the scattering length for which an Efimov state is at the atom-dimer threshold. We measure energies relative to the 3 -atom threshold, so the atom-dimer threshold is $\left|E_{3}\right|=\left|E_{2}\right|$. The parameter $a_{*}$ is related to the 3-body parameter $\Lambda_{*}$ introduced in Ref. 12 by $s_{0} \ln \left(a_{*} \Lambda_{*}\right)=1.44 \bmod \pi$, where $s_{0}=1.00624$. The Efimov spectrum is shown in Fig. [1 If the scattering length is further increased to $22.7 a_{*}$, the binding energy of the first Efimov trimer becomes $\left|E_{3}\right|=6.8\left|E_{2}\right|$ and a second Efimov trimer appears at the atom-dimer threshold $\left|E_{3}\right|=\left|E_{2}\right|$. Efimov theory predicts that if $a$ is large, the next critical value $a_{*}$ lies between $a$ and $22.7 a$. For $a>0$, large scattering length can be defined by the condition that $\left|E_{2}\right|$ be given by (11). For the experiment of Ref. [10], this is satisfied to within $20 \%$ at $a=925 a_{0}$ $(B=158.5 \mathrm{G})$ and it becomes increasingly accurate as $B$ approaches the resonance. Thus a reasonable range for the 3 -body parameter is $925 a_{0}<a_{*}<21000 a_{0}$. The 
value of $a_{*}$ depends exponentially on an angle that is determined by short-distance aspects of the 3-body problem. If we assume a uniform probability distribution for this angle, there is a $50 \%$ probability that $a_{*}<4400 a_{0}$.

If $a$ is close enough to $a_{*}$, atom-dimer scattering will be dominated by the Efimov state near threshold. The atom-dimer scattering length is [4, 12, 13]

$$
a_{12}=\left[1.46+2.15 \cot \left(s_{0} \ln \left(a / a_{*}\right)\right)\right] a .
$$

It diverges as $a \rightarrow a_{*}$, where the Efimov state is at the atom-dimer threshold. If $a$ is just above $a_{*}$, we can approximate $E_{3}-E_{2}$ by $-\hbar^{2} /\left(2 m_{12} a_{12}^{2}\right)$, where $m_{12}=2 m / 3$ is the reduced mass of the atom and the dimer. As $a \rightarrow a_{*}$, the difference in binding energies approaches

$$
\left|E_{3}-E_{2}\right| \approx 0.16 \ln ^{2}\left(a / a_{*}\right) \hbar^{2} /\left(m a^{2}\right) \quad\left(a>a_{*}\right) .
$$

This expression underestimates $\left|E_{3}-E_{2}\right|$ by $20 \%$ at $a=$ $3.3 a_{*}$ and by a factor of 4 at $a=22.7 a_{*}$. A more accurate approximation can easily be obtained by the numerical solution of the equation given in Ref. 14]. If $a$ decreases below $a_{*}$, the Efimov state disappears from the spectrum.

If the number densities $n_{1}$ and $n_{2}$ of the atom and dimer condensates are sufficiently large, it may be necessary to take into account mean-field energy shifts. The mean-field energy of an atom includes the terms $2 \pi \hbar^{2} a n_{1} / m$ and $3 \pi \hbar^{2} a_{12} n_{2} / 2 m$, while that of a dimer includes a term $3 \pi \hbar^{2} a_{12} n_{1} / 2 m$. The mean-field energy of the trimer and the term in the mean-field energy of a dimer proportional to $n_{2}$ are determined by solutions to the $n$-body problem with $n \geq 4$ and are not known.

The simplest way to observe the trimer condensate is to choose the magnetic field during the hold time $\tau$ so that $a_{\text {hold }}>a_{*}$, such as the dotted line in Fig. 1 In this case, there will be 3 coexisting condensates during step 2 . The first pulse will transfer atoms coherently from the atom condensate into dimer and trimer condensates. During the hold time, the dimer and trimer condensates will accumulate phases relative to the atom condensate of $2 \pi \nu_{2} \tau$ and $2 \pi \nu_{3} \tau$, respectively, where $\nu_{3}=\left|E_{3}^{\text {hold }}\right| / 2 \pi \hbar$ and $\left|E_{3}^{\text {hold }}\right|$ is the trimer binding energy when the scattering length is $a_{\text {hold }}$. The second pulse will transfer atoms coherently between the three condensates. After the second pulse, which decreases the scattering length to $a_{\text {final }}<a_{*}$, the trimer condensate will have disappeared, but it will have left behind a smoking gun. The numbers $N_{\text {remnant }}, N_{\text {burst }}$ and $N_{\text {missing }}$ will exhibit oscillations in $\tau$ with frequency $\nu_{3}-\nu_{2}$ on top of the oscillations with frequency $\nu_{2}$.

It could be difficult to increase $a_{\text {hold }}$ above $a_{*}$, because 3-body loss rates during the hold time increase like $a_{\text {hold }}^{4}$. It would be much easier to increase $a_{\max }$ above $a_{*}$. If $a_{\text {hold }}<a_{*}<a_{\text {max }}$, a trimer condensate could be created during the pulses. But after each pulse, the trimer would be unstable and would quickly decay into an energetic dimer and an atom. This would destroy all information about the angle $2 \pi \nu_{3} \tau$, so the numbers $N_{\text {remnant }}, N_{\text {burst }}$ and $N_{\text {missing }}$ would oscillate only with frequency $\nu_{2}$. The lowest oscillation frequency observed in the experiment of Ref. [10] was $10 \mathrm{kHz}$, which corresponds to $a_{\text {hold }} \approx$ $2000 a_{0}$. If a second oscillation frequency in this data could be excluded, it would place an experimental lower bound $a_{*}>2000 a_{0}$.

It might be possible to find indirect evidence for the trimer condensate by studying the bursts of atoms created in experiments with a single pulse that reaches a maximum $a_{\max }>a_{*}$, such as the dashed line in Fig. [1] The time sequence $a(t)$ would consist of a ramp from $a_{\text {init }}$ to $a_{\max }$, a holding time, and then a ramp down to $a_{\text {final }}$. The ramp up to $a_{\max }$ would allow the coherent transfer of atoms from the atom condensate into dimer and trimer condensates. The number of dimers and trimers in these condensates would depend on the ramp rate, and could therefore be varied. If the ramp down to $a_{\text {final }}$ is sufficiently rapid, it would create a burst of atoms with 2 components, one from the dimer condensate and one from the trimer condensates.

We first consider the burst of atoms emitted by the dimer condensate. If the scattering length is changed suddenly from one large value $a$ to another $a^{\prime}$, the probabilities for the final states are determined by the overlaps of the initial and final wavefunctions. The wavefunction for a dimer at rest is proportional to $e^{-r / a} / r$. The wavefunction for a scattering state consisting of two atoms with wavevectors $\pm \mathbf{k}$ is $\cos (\mathbf{k} \cdot \mathbf{r})-(1 / a+i k)^{-1} e^{i k r} / r$. The probability that the dimer remains a dimer is

$$
\begin{aligned}
P[D \rightarrow D] & =4 a a^{\prime} /\left(a+a^{\prime}\right)^{2} & & a^{\prime}>0, \\
& =0 & & a^{\prime}<0 .
\end{aligned}
$$

The probability density for the dimer to become a pair of atoms with wavevectors $\pm \mathbf{k}$ is

$$
d P[D \rightarrow A A]=\frac{\left(a-a^{\prime}\right)^{2} /\left(\pi^{2} a^{3} a^{\prime 2}\right)}{\left(k^{2}+1 / a^{2}\right)^{2}\left(k^{2}+1 / a^{\prime 2}\right)} d^{3} k .
$$

The distribution of the energy $E=\hbar^{2} k^{2} / 2 m$ of the atoms in the burst is obtained by multiplying (5) by $2 N_{D}$, where $N_{D}$ is the number of dimers in the dimer condensate before the change in $a$. The mean energy is $\langle E\rangle=(2 a+$ $\left.\left|a^{\prime}\right|\right) /\left(2\left|a^{\prime}\right|\right)\left|E_{2}\right|$, where $\left|E_{2}\right|=\hbar^{2} /\left(m a^{2}\right)$.

We now consider the burst emitted by a trimer condensate. Determining the probabilities for the final states in general requires solving a nontrivial 3-body problem. However, as $a \rightarrow a_{*}$, the 3-body problem reduces to the 2 -body problem of an atom and dimer with resonant interactions. Therefore if $a$ and $a^{\prime}$ are both close to $a_{*}$, we can apply the analogs of (4) and (5). The probability $P[T \rightarrow T]$ for the trimer to remain a trimer is given by (4I) with $a \rightarrow a_{12}$ and $a^{\prime} \rightarrow a_{12}^{\prime}$. The probability density $d P[T \rightarrow A D]$ for a trimer to become an atom and a dimer with wavenumbers $\pm \mathbf{k}$ is given by (15) with 


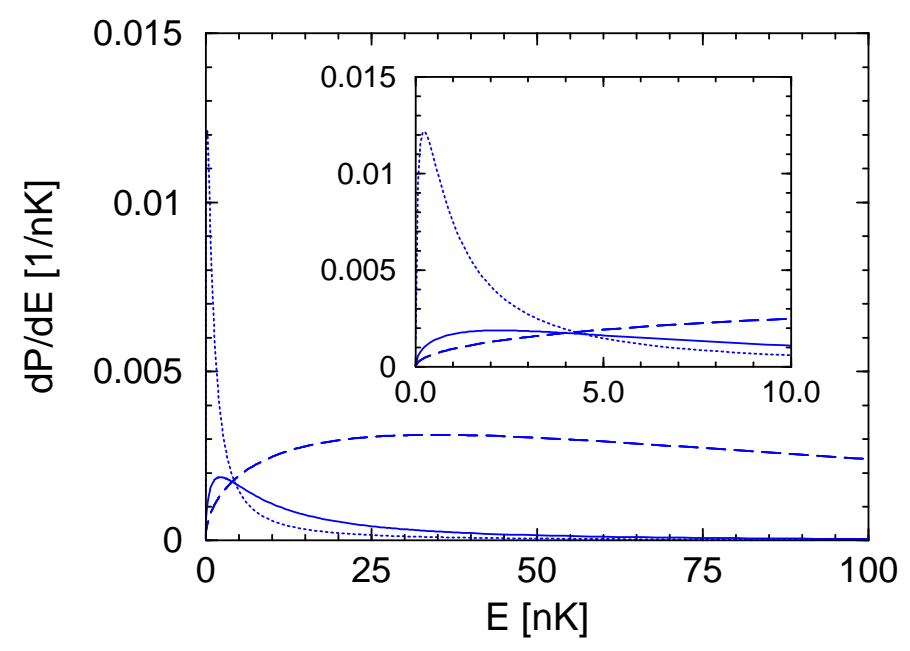

FIG. 2: The energy distributions of the burst atoms emitted when the scattering length is suddenly changed from $a=$ $3000 a_{0}$ to $1000 a_{0}$. The dashed line is the distribution of atoms emitted by the dimer condensate normalized to 1 . The solid (dotted) line is the distribution of atoms emitted by the trimer condensate for $a_{*}=1500 a_{0}\left(2500 a_{0}\right)$ normalized to $1 / 25$. The inset shows the low-energy region in more detail.

$a \rightarrow a_{12}$ and $a^{\prime} \rightarrow a_{12}^{\prime}$. The distribution of the energy of the atoms in the burst is obtained by multiplying by $N_{T}$, where $N_{T}$ is the number of trimers in the trimer condensate before the change in $a$. The mean energy of the atoms is $\langle E\rangle=\left(2 a_{12}+\left|a_{12}^{\prime}\right|\right) /\left(2\left|a_{12}^{\prime}\right|\right)\left|E_{3}-E_{2}\right|$, where $\left|E_{3}-E_{2}\right|$ is given in (3). In Fig. 22 we show the shapes of the energy distributions $d P / d E=(k / 2 E) d P / d k$ of the burst atoms created if the scattering length is suddenly changed from $a=3000 a_{0}$ to $1000 a_{0}$. The correct normalizations are obtained by multiplying by $2 N_{D}$ for the dimer condensate and by $25 N_{T}$ for the trimer condensate.

If a method can be developed to image the dimers, it could provide further evidence for a trimer condensate. If $a_{\max }<a_{*}$, most of the dimers should be in the dimer condensate. However if $a_{\max }>a_{*}$, there should also be a burst of dimers whose distribution in the energy $E=\hbar^{2} k^{2} / 4 m$ is given by (15) with $a \rightarrow a_{12}, a^{\prime} \rightarrow a_{12}^{\prime}$, and normalization $N_{T}$. This burst of dimers would be another signature of the trimer condensate.

If the atom-atom scattering length $a$ is large, other 3body observables are completely determined by $a$ and the 3 -body parameter $a_{*}$. Once $a_{*}$ is determined, one may be able to test the remarkable prediction of an approximate zero in the 3-body recombination rate into an atom and the shallow dimer [15]. The rate per unit time and volume of 3-body recombination events in a BEC is proportional to the cube of the number density: $\nu=\alpha n^{3} / 6$. The contribution to $\alpha$ from recombination into the shallow dimer is 13

$$
\alpha=67.1 \sin ^{2}\left[s_{0} \ln \left(a / a_{*}\right)+1.63\right] \hbar a^{4} / m,
$$

which vanishes at $a=0.20 a_{*}, 4.5 a_{*}, \ldots$ The contributions to $\alpha$ from recombination into deeply-bound diatomic molecules also scale like $a^{4}$, so the zeroes in (6) should be interpreted as local minima of $\alpha / a^{4}$.

There is a qualitative difference between the Efimov states for heavy alkali atoms such as ${ }^{85} \mathrm{Rb}$ near a Feshbach resonance and those for ${ }^{4} \mathrm{He}$. In the case of ${ }^{85} \mathrm{Rb}$, the 2-body bound states include more deeply-bound diatomic molecules as well as the shallow dimer. The Efimov states have a width, because they can decay into an atom and deeply-bound molecules. There are no reliable estimates of the widths of the Efimov states. These states are of physical relevance only if their widths are small compared to their binding energies. The observation of a condensate of Efimov trimers would provide dramatic confirmation of the importance of Efimov states in the physics of cold atoms with resonant interactions. It would also provide a glimpse into the rich and beautiful physics of the resonant BEC.

This research was supported in part by DOE grant DEFG02-91-ER4069 and by NSF grant PHY-0098645. We thank Neil Claussen, Eric Cornell, Jason Ho, and Erich Mueller for useful discussions.

[1] A. Einstein, Sitzungsb. Kgl. Preuss. Akad. Wiss. 23, 3 (1925).

[2] M.H. Anderson et al., Science 269, 198 (1995); C.C. Bradley et al., Phys. Rev. Lett. 75, 1687 (1995); K.B. Davis et al., Phys. Rev. Lett. 75, 3969 (1995).

[3] V.N. Efimov, Sov. J. Nucl. Phys. 12, 589 (1971); 29, 546 (1979); Nucl. Phys. A 210, 157 (1973).

[4] E. Braaten and H.-W. Hammer, Phys. Rev. A 67, 042706 (2003), and references therein.

[5] A. Bulgac, Phys. Rev. Lett. 89, 050402 (2002).

[6] B.J. Cusack et al., Phys. Rev. A 65, 013609 (2002).

[7] E. Tiesinga, B.J. Verhaar, and H.T.C. Stoof, Phys. Rev. A 47, 4114 (1993); A.J. Moerdijk, B.J. Verhaar, and A. Axelsson, Phys. Rev. A 51, 4852 (1995).

[8] S. Inouye et al., Nature 392, 151 (1998); J. Stenger et al., Phys. Rev. Lett. 82, 2422 (1999).

[9] S.L. Cornish et al., Phys. Rev. Lett. 85, 1795 (2000); J.L. Roberts et al., Phys. Rev. Lett. 86, 4211 (2000); N.R. Claussen et al., Phys. Rev. Lett. 89, 010401 (2002).

[10] E.A. Donley et al., Nature 417, 529 (2002).

[11] S.J.J.M.F. Kokkelmans and M.J. Holland, Phys. Rev. Lett. 89, 180401 (2002).

[12] P.F. Bedaque, H.-W. Hammer, and U. van Kolck, Phys. Rev. Lett. 82, 463 (1999); Nucl. Phys. A 646, 444 (1999).

[13] E. Braaten and H.-W. Hammer, Phys. Rev. Lett. 87, 160407 (2001); P.F. Bedaque, E. Braaten, and H.W. Hammer, Phys. Rev. Lett. 85, 908 (2000).

[14] E. Braaten, H.-W. Hammer, and M. Kusunoki, Phys. Rev. A 67, 022505 (2003).

[15] B.D. Esry, C.H. Greene, and J.P. Burke, Phys. Rev. Lett. 83, 1751 (1999); E. Nielsen and J.H. Macek, Phys. Rev. Lett. 83, 1566 (1999). 